\title{
An overview of diffusion models for intracellular dynamics analysis
}

\author{
Vincent Briane, Myriam Vimond, Charles Kervrann* \\ * Corresponding author: Charles Kervrann,Inria, Centre Rennes-Bretagne Atlantique, SERPICO Project Team, 35042 Rennes, France. Tel.: + 332998422 \\ 21; Fax: +33 2998471 71; E-mail: charles.kervrann@inria.fr
}

\begin{abstract}
We present an overview of diffusion models commonly used for quantifying the dynamics of intracellular particles (e.g. biomolecules) inside eukaryotic living cells. It is established that inference on the modes of mobility of molecules is central in cell biology since it reflects interactions between structures and determines functions of biomolecules in the cell. In that context, Brownian motion is a key component in short distance transportation (e.g. connectivity for signal transduction). Another dynamical process that has been heavily studied in the past decade is the motor-mediated transport (e.g. dynein, kinesin and myosin) of molecules. Primarily supported by actin filament and microtubule network, it ensures spatial organization and temporal synchronization in the intracellular mechanisms and structures. Nevertheless, the complexity of internal structures and molecular processes in the living cell influence the molecular dynamics and prevent the systematic application of pure Brownian or directed motion modeling. On the one hand, cytoskeleton density will hinder the free displacement of the particle, a phenomenon called subdiffusion. On the other hand, the cytoskeleton elasticity combined with thermal bending can contribute a phenomenon called superdiffusion. This paper discusses the basics of diffusion modes observed in eukariotic cells, by introducing the essential properties of these processes. Applications of diffusion models include protein trafficking and transport and membrane diffusion.
\end{abstract}

Key words: diffusion; Brownian motion; stochastic models; intracellular dynamics; microscopy.

\section{Introduction}

As the interior of a eukaryotic living cell is a fluctuating environment, we model the trajectories of particles with stochastic processes with continuous paths. Diffusions belong to this class of processes and can model a large range of intracellular movements. In what follows, we focus on diffusion defined as solutions of a stochastic differential equation (SDE) and we study stochastic models used in biophysics that generalize Brownian motion.

In the literature [1, 2], biophysicians distinguish four main types of diffusions, namely Brownian motion (also referred to as free diffusion), superdiffusion, confined diffusion and anomalous diffusion. Trajectories illustrating these four types of diffusion are represented in Figure 1. These different diffusions correspond to specific biological scenarios. If a particle evolves freely inside the cytosol or along the plasma membrane, motion is modeled by free diffusion and is due to the constant collisions with smaller particles animated by thermal fluctuations. Hence, the particle does not travel along any particular direction and can take a very long time to go to a precise area in the cell. Active intracellular transport can overcome this difficulty so that motion is faster and direct specific. The particles (called in this context cargo) are carried by molecular motors along the

Vincent Briane is a PhD student at Inria, Centre Rennes-Bretagne Atlantique (SERPICO Project Team) and at CREST (Ensai, Université Bretagne Loire), Bruz, France.

Myriam Vimond is an Assistant Professor in Statistics at CREST (Ensai, Université Bretagne Loire), Bruz, France. Charles Kervrann is an Inria Research Director and is head of Serpico Team, Centre Rennes-Bretagne Atlantique (SERPICO Project Team), Rennes, France. 


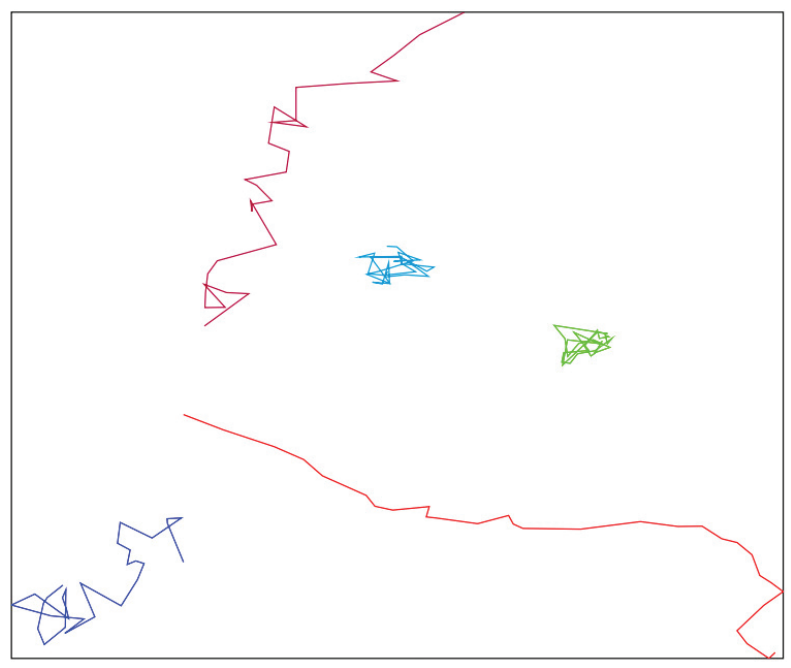

Figure 1. Representative trajectories from simulated data. The blue trajectory is Brownian; the purple trajectory is from a Brownian motion with drift (67) and illustrates superdiffusion; the red trajectory is from an $\mathrm{fBm}$ (22) (parameter $\mathfrak{h}>1 / 2$ ) and illustrates superdiffusion; the cyan trajectory is from an OrnsteinUhlenbeck process (62) and illustrates confined diffusion; the green trajectory is from an $\mathrm{fBm}(22)(\mathfrak{h}<1 / 2)$ and illustrates anomalous diffusion.

cytoskeleton meshwork. Superdiffusions model the motion of molecular motors and their cargo in the viscoelastic cytosol of living cells [4]. Confined or restricted diffusion [5, 6] is characteristic of particles that either bind to a specific site for a period of time before dissociating and moving away or are trapped in subdomains such as cytoskeleton 'cages'. Anomalous diffusion includes particles which encounters dynamic or fixed obstacles $[7,8]$ or particles slowed by the contrary current due to the viscoelastic properties [9] of the cytoplasm. A classification of protein trajectories into the three types of diffusion (Brownian, superdiffusion and subdiffusion) is shown in Figure 2. This classification is obtained with our three-decision test procedure described in [3].

\section{Mean square displacement}

In biophysics, the different types of diffusions are characterized by the mean square displacement (MSD) [1]. Given a particle trajectory $\left(X_{t}\right)_{t>0}$, the MSD is defined as the function

$$
\operatorname{MSD}(t)=\mathbb{E}\left(\left\|X_{t+t_{0}}-X_{t_{0}}\right\|^{2}\right),
$$

where $\|\cdot\|$ is the Euclidean norm and $\mathbb{E}$ is the expectation of the probability space. The MSD function of Brownian motion is linear $(\operatorname{MSD}(t) \propto t)$, while the MSD function of subdiffusion (respectively superdiffusion) grows slower (respectively faster) than the linear function.

This property makes the MSD a popular criterion to analyze intracellular motion as Brownian motion is the process of reference. The typical MSD curves of the different diffusion models are represented in Figure 3. In practical imaging, we observe the successive positions of a single particle $X_{t_{0}}, X_{t_{1}}, \ldots, X_{t_{n}}$ in the two or three dimensions at equidistant times, that is $t_{i+1}-t_{i}=\Delta$.

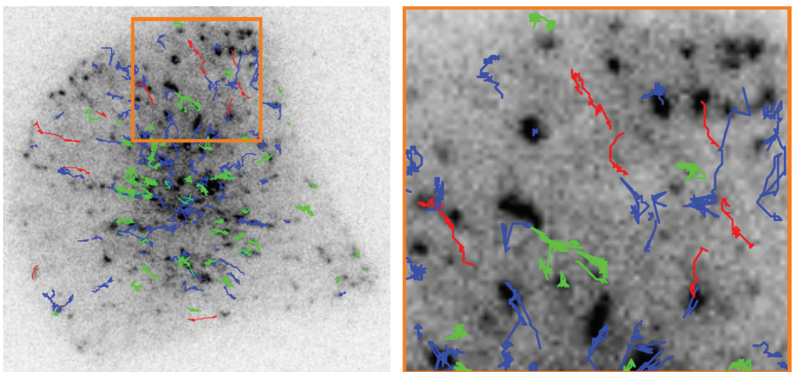

Figure 2. Classification of two-dimensional trajectories from the Rab11 protein sequence in a single cell observed in TIRF microscopy (courtesy of UMR 144 CNRS Institut Curie PICT IBiSA). We use the three-decision test procedure developed in [3] at level $\alpha=5 \%$. The Brownian trajectories are in blue, the subdiffusive trajectories in green and the superdiffusive trajectories in red.

\section{The MSD is estimated at lag $j$ by}

$$
\widehat{\operatorname{MSD}}(j \Delta)=\frac{1}{n-j+1} \sum_{k=0}^{n-j}\left\|X_{t_{k+j}}-X_{t_{k}}\right\|^{2}
$$

Computing the estimator (2) at different lagj gives an estimation of the MSD function (1). Then the simplest rule to classify a trajectory is based on a fit of the MSD function (1) to $t \rightarrow t^{\beta}$. Feder et al. [10] states that the trajectory is subdiffusive if $\beta<0.9$, superdiffusive if $\beta>1.1$ and Brownian if $0.9<\beta<1$. If $\beta<0.1$ it states that the particle does not move, see Figure 4.

\section{Limitations}

First, the MSD function is a summary measure based on a 2nd order moment and is not sufficient to characterize the dynamics of the trajectory. Accordingly, several authors (e.g. [11, 12]) proposed other statistics which can be associated with MSD for trajectory analysis. Lund et al. [13] propose a decision tree for selection motion model combining MSD, Bayesian information criterion and the radius of gyration. Lysy et al. [14] present a likelihood-based inference as an alternative to MSD for the comparison between two models of subdiffusions: fractional Brownian motion ( $\mathrm{fBm}$ ) and a generalized Langevin equation (GLE).

Secondly, the estimation of the MSD function (1) is tricky as the variance of estimator (2) increases with the time lag. Figure 4 illustrates this problem in the case of Brownian trajectories. It suggests that the classification of Feder et al. [10] based on parameter $\beta$ overdetects subdiffusion and superdiffusion while it is Brownian motion. It is worth noting that MSD can vary with the time scale (confined diffusion or diffusion with obstacles is Brownian diffusion at short time scale but exhibits a MSD exponent $<1$ at longer time scale).

Moreover, the MSD variance is also severely affected at short time lags by dynamic localization error and motion blur. Michalet [15] details an iterative method, known as the Optimal Least Square Fit for determining the optimal number of points to obtain the best fit to MSD in the presence of localization uncertainty. In order to take account of the variance of the MSD estimate, several authors use a set of independent trajectories rather than single trajectories. These trajectories may have different lengths but are assumed to have the same kind of motion. For instance, Pisarev et al. [16] consider weighted-least-square 


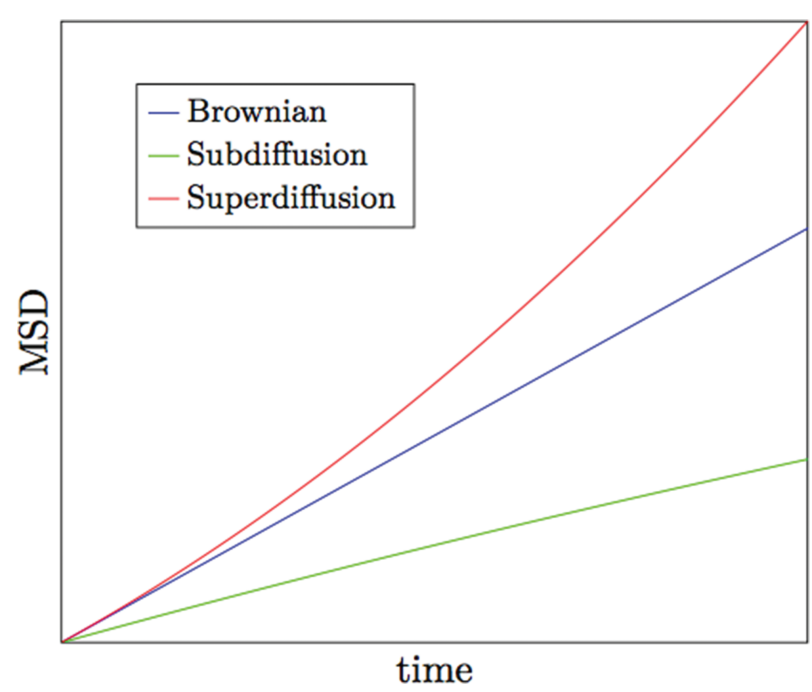

Figure 3. Typical MSD curves of the different diffusion types.

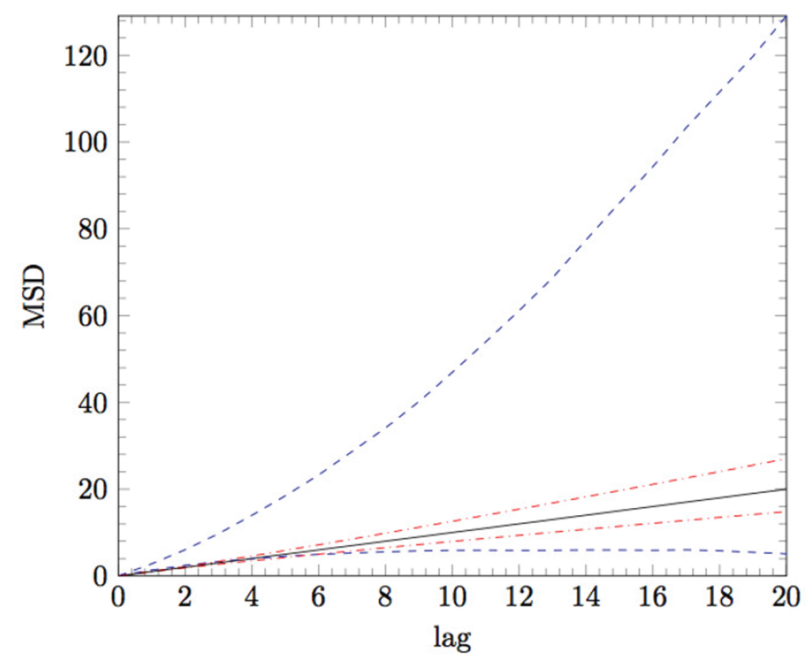

Figure 4. A classification rule for motion modes from MSD. The dashdotted lines are the bound defined by [10], $t \rightarrow t^{\beta}, \beta=0.9$ and 1.1. The dashed lines are the pointwise high probability interval of $95 \%$ associated to the empirical MSD curve for a standard Brownian motion trajectory of length $n=30$. The bounds of the interval are the $2.5 \%$ and $97.5 \%$ empirical quantile of (2) and are computed by Monte Carlo simulation from 10001 Brownian trajectories of length $n=30$.

estimate for $\beta$ by estimating the variance of pathwise MSD. Their motion model selection is then based on the modified Akaike's information criterion. Monnier et al. [17] propose a Bayesian approach to compute relative probabilities of an arbitrary set of motion models (free, confined, anomalous or directed diffusion). In general, this averaging process can lead to oversimplication and misleading conclusions about the biological process [12].

Finally, it is hard to interpret the MSD function. At short time scale the MSD function of confined/anomalous diffusion is approximately linear, which is characteristic of Brownian motion and only exhibits a MSD exponent $<1$ at longer time scale. Moreover, the MSD function is not a reliable tool in case the particle switches motion over time.

\section{Paper organization}

The remainder of the paper is organized as follows. In the next section, we present the probabilistic tools in order to define diffusion processes. Such processes are of great importance for modeling intracellular dynamics. To this end, we focus on $d$-dimensional processes with $d=2$ or $d=3$. In Diffusion for Modeling Intracellular Trajectories section, we present the three main types of diffusion studied in biophysics to model intracellular motion, namely Brownian motion, subdiffusion and superdiffusion. We also described the different biological scenarios associated with each mode of diffusion.

In this paper, we compute the theoretical MSD (1) for each presented motion model and classify it as Brownian, superdiffusion or subdiffusion accordingly. However (and even if it is out of the scope of this paper), we must emphasize that MSD has some limitations.

\section{Stochastic processes, Brownian motion and diffusions}

It is worth noting that the biophysics literature uses the word diffusion in a very broad sense [18]. Here we introduce the probabilistic concept of diffusion presented in [19] and [20]. First, we define the notion of stochastic processes. Then, we put an emphasis on Brownian motion, the cornerstone process which allows to build all the diffusion processes. We describe diffusion processes driven by Brownian motion. Finally, we deal with an extension of Brownian motion, namely $\mathrm{fBm}$ [21]; we present quickly diffusion processes driven by $\mathrm{fBm}$.

\section{Stochastic process}

Let $(\Omega, \mathcal{F}, P)$ a probability space where $\Omega$ is the sample space, $\mathcal{F}$ a field and $P$ a probability measure. A d-dimensional 'stochastic process' is a function

$$
\begin{aligned}
I \times \Omega & \rightarrow \mathbb{R}^{d} \\
(t, \omega) & \mapsto X(t, \omega),
\end{aligned}
$$

where $I$ is a time interval. We note this application $\left(X_{t}\right)_{t \in I}$ or simply $\left(X_{t}\right)$. We present briefly stochastic processes from two angles.

Let $t \in I$, the application,

$$
\begin{aligned}
& \Omega \rightarrow \mathbb{R}^{d} \\
& \omega \mapsto X(t, \omega)
\end{aligned}
$$

is the random state of the process at time $t$. It is a random variable defined on $(\Omega, \mathcal{F}, P)$. Then a stochastic process can be seen as the collection of random variables $\{\omega \mapsto X(t, \omega), t \in I\}$.

Let $\omega \in \Omega$, the application

$$
\begin{aligned}
I & \rightarrow \mathbb{R}^{d} \\
t & \mapsto X(t, \omega)
\end{aligned}
$$

is called a 'trajectory' or a 'path' of the stochastic process $\left(X_{t}\right)_{t \in I}$. A stochastic process may be seen as an application from $\Omega$ to the set of functions from $I=[0, T]$ to $\mathbb{R}^{d}$. As previously mentioned, we consider only the stochastic processes whose trajectories are continuous, that is $t \rightarrow X_{t}(\omega)$ is continuous for almost $\omega \in \Omega$. 


\section{Finite-dimensional distribution}

A stochastic process may be seen as a random variable from $(\Omega, \mathcal{F}, P)$ to the measurable space

$$
\left(\mathfrak{F}\left([0, T], \mathbb{R}^{d}\right), \otimes_{\mathrm{t} \in[0, T]} \mathcal{B}_{d}\right),
$$

where $\mathfrak{F}\left([0, T], \mathbb{R}^{d}\right)$ is the set of functions from $[0, T]$ to $\mathbb{R}^{d}, \mathcal{B}_{d}$ is the Borelian sigma-algebra and $\otimes_{\mathrm{t} \in[0, \mathrm{~T}]} \mathcal{B}_{d}$ is the sigma-algebra generated by all the finite-dimensional cylindrical sets of $\mathfrak{F}\left([0, T], \mathbb{R}^{d}\right)$. Then the stochastic process $X$ induces a probability measure on $\left(\mathfrak{F}\left([0, T], \mathbb{R}^{d}\right), \otimes_{t \in[0, T]} \mathcal{B}_{d}\right)$ which is defined through the finitedimensional distribution.

Now we define the concept of finite-dimensional distribution. Let $J=\left\{t_{0}, t_{1}, \ldots, t_{n}\right\}$ such that $t_{i} \in I$ and $t_{0}<t_{1}<, \ldots,<t_{n}$. We note,

$$
X_{J}=\left(X_{t_{0}}, \ldots, X_{t_{n}}\right)
$$

the random vector whose components $X_{t_{i}} \in \mathbb{R}^{d}$. The distribution $\mu_{\mathrm{J}}$ of $\mathrm{X}_{\mathrm{J}}$ is the joint distribution

$$
\mu_{\mathrm{J}}(\mathrm{A})=\mathrm{P}\left(\mathrm{X}_{\mathrm{t}_{0}} \in \mathrm{A}_{0}, \ldots, \mathrm{X}_{\mathrm{t}_{n}} \in \mathrm{A}_{n}\right)
$$

where $A_{i} \in \mathbb{R}^{d}$ and $A=A_{0} \times \cdots \times A_{n}$.

The 'finite-dimensional distributions' of $X$ is the family of distributions $\left\{\mu_{j} \mid J\right.$ a finite set of $\left.I\right\}$. If the finite-dimensional distributions $\mu_{\text {J }}$ satisfy a technical criterion called consistency then the Kolmogorov extension theorem guarantees the existence of a stochastic process $\mathrm{X}$ with finite-dimensional distributions $\mu_{\mathrm{J}}$ on $(\Omega, \mathcal{F}, P)[22$, Chapter 1 , Section 1.1].

\section{Filtered probability space}

We state previously that a stochastic process can be seen as a collection of random variables defined on $(\Omega, \mathcal{F}, P)$. More precisely the random variable (4) is defined on $\left(\Omega, \mathcal{F}_{t}, P\right)$ where $\mathcal{F}_{t} \subset \mathcal{F}$. This reflects that the outcome of the random variable (4) depends on what happened before $t$, that is on the historic of the process until time $t$.

Then we define the concept of filtration. A 'filtration' $\mathbb{F}$ is a family $\left(\mathcal{F}_{\mathrm{t}}\right.$ ) of increasing fields on $(\Omega, \mathcal{F})$ that is $\mathcal{F}_{\mathrm{s}} \subset \mathcal{F}_{\mathrm{t}} \subset \mathcal{F}$ for $\mathrm{s}<\mathrm{t}$. $\mathbb{F}$ specifies how the information is revealed over time. The property that a filtration is increasing corresponds to the fact the information is not forgotten. Finally, a stochastic process $\mathrm{X}$ is called adapted to a filtration $\mathbb{F}$ if, for all t, the random variable $\omega \mapsto X(t, \omega)$ is $\mathcal{F}_{t}$-measurable.

\section{Brownian motion}

The observation of the erratic motion of a pollen particle suspended in a fluid by the botanist R. Brown in 1828 marks the 1st step in the development of the Brownian motion theory. In 1905, Einstein argued that the movement of the particle is due to its bombardment by the particles of the fluid; he obtained the equations of Brownian motion. The underlying probability theory was derived by N. Wiener in 1923 that is why Brownian motion is also known as the Wiener process. In this section, we define the one-dimensional Brownian motion and characterize it as a Gaussian process. Then, we define the d-dimensional Brownian motion.

\section{Definition}

The 'one-dimensional Brownian motion' $\left(B_{t}\right)$ is a stochastic process with the following properties:

- $\left(B_{t}\right)$ is a process with 'independents increments'. For all $t>s$, $B_{t}-B_{s}$ is independent of the field $\mathcal{F}_{s}$ generated by the historic of the process $\left(B_{u}\right)_{u \in[0, s]}$ until the time $s$.

- For all $t>s, B_{t}-B_{s}$ has normal distribution with mean 0 and variance $t-s$.

- The paths of $\left(B_{t}\right)$ are almost surely continuous.

\section{Gaussian process}

A 'Gaussian process' is a process for which all the finitedimensional distributions are multivariate normal. We have the following theorem.

Theorem 2.1. A Brownian motion started at zeros is a Gaussian process with zero mean and covariance function $\min (t, s)$. Conversely, a Gaussian process with zero mean and covariance $\min (t, s)$ is a Brownian motion.

\section{Multivariate Brownian motion}

As we already stated, we are interested in modeling the trajectories of particle in dimensions 2 and 3. We define the ' $d$ dimensional Brownian motion' $(d \geq 1)$ as the random vector $B_{t}=\left(B_{t}^{1}, \ldots, B_{t}^{d}\right)$ where all coordinates $B_{t}^{i}$ are independent onedimensional Brownian motions.

\section{Diffusion process}

We present briefly the family of stochastic processes of interest in this paper, namely the diffusion processes. First, we recall the Markov property which is a central notion for defining the diffusion processes. Then we give the definition of diffusions and some characterizations of these processes.

\section{Markov property}

The Markov property states that if we know the present state of the process, the future behavior of the process is independent of its past. For instance, a simple model of weather forecast assumes that the probability to have rain at day $j$ given the information of the weather on the previous days is the same as the probability to have rain at day $j$ given the restricted information of the weather at day $j-1$. Let note $\left(X_{i}\right)$ the process giving the weather at each day $i$ and note $k$ the modality corresponding to rain. In this discrete set-up, the Markov property can be written as

$$
P\left(X_{j}=k \mid X_{j-1}, \ldots, X_{0}\right)=P\left(X_{j}=k \mid X_{j-1}\right) .
$$

As we work with stochastic processes defined continuously in time, the historic of the process given by $X_{j-1}, \ldots, X_{0}$ in the discrete case is replaced by the field $\mathcal{F}_{t}$ at time t. Then a $d-$ dimensional continuous stochastic process $\left(X_{t}\right)$ is Markovian if

$$
P\left(X_{t+s} \in A \mid \mathcal{F}_{t}\right)=P\left(X_{t+s} \in A \mid X_{t}\right),
$$

where $A \in \mathbb{R}^{d}$. Then we have the following theorem.

Theorem 2.2. The Brownian motion $\left(B_{t}\right)$ has the Markov property. 
Remark 2.1. Another difference (apart from the conditioning) between Equations (8) and (9) is the different nature of the events $\left\{X_{j}=k\right\}$ and $\left\{X_{t+s} \in A\right\}$. It is due to the fact that in Equation (8) the state space of the stochastic process (modality of weather) is countable while the state space of the stochastic process is the whole space $\mathbb{R}^{d}$ (not countable) in (9).

\section{Diffusions}

A 'diffusion process' $\left(X_{t}\right)$ is a continuous time process which possesses the Markov property and for which the sample paths are continuous. Moreover, every diffusion process satisfies three key conditions see [19, Chapter 15, Section 1]. The 1st condition states that large displacements of magnitude exceeding $\epsilon>0$ are very unlikely over sufficiently small intervals

$$
\lim _{\Delta \rightarrow 0} \frac{1}{\Delta} P\left(\left\|X_{t+\Delta}-X_{t}\right\|>\epsilon \mid X_{t}=x\right)=0, \quad \forall \epsilon>0, \quad \forall x \in \mathbb{R}^{d}
$$

where $\|\cdot\|$ denotes the Euclidean norm. In other words, condition (10) prevents the diffusion process from having discontinuous jumps. The two last conditions characterize the mean and the variance of the infinitesimal displacements and affirm the existence of the limits

$$
\begin{gathered}
\lim _{\Delta \rightarrow 0} \mathbb{E}\left(X_{t+\Delta}-X_{t} \mid X_{t}=x\right)=\mu(x, t), \quad \forall x \in \mathbb{R}^{d}, \\
\lim _{\Delta \rightarrow 0} \mathbb{E}\left(\left(X_{t+\Delta}-X_{t}\right)\left(X_{t+\Delta}-X_{t}\right)^{\top} \mid X_{t}=x\right)=\sigma^{2}(x, t), \quad \forall x \in \mathbb{R}^{d},
\end{gathered}
$$

where $T$ denotes the transpose operator; $\mu(x, t): \mathbb{R}^{d} \times \mathbb{R}^{+} \rightarrow \mathbb{R}^{d}$ is the drift parameter; $\sigma^{2}(x, t): \mathbb{R}^{d} \times \mathbb{R}^{+} \rightarrow S_{+}^{d}$ is the diffusion coefficient where $S_{+}^{d}$ is the set of positive semi-definite matrix of size $d$.

In particular, Brownian motion is a diffusion process: its drift is the null function, and its diffusion coefficient is constant.

\section{Stochastic differential equation}

The most common approach for defining diffusion processes is to see them as the solution of SDEs.

\section{Physical model}

Initially diffusion models were developed to describe the motion of a particle in a fluid submitted to a deterministic force due to the fluid and a random force due to random collisions with other particles. That is why we model efficiently the motion of intracellular particles with diffusion. Let $X_{t} \in \mathbb{R}^{d}$ be the position of the particle at time $t$ and $\left(B_{t}\right)$ a d-dimensional Brownian motion; assume that $X_{t}=x$. Then the displacement of the particle between $t$ and $t+\Delta$ is approximately given by

$$
X_{t+\Delta}-x \approx \mu(x, t) \Delta+\sigma(x, t)\left(B_{t+\Delta}-B_{t}\right)
$$

The component $\mu(x, t) \Delta$ is the displacement due to the fluid where the velocity of the fluid is given by the drift $\mu(x, t)$. The term $\sigma(x, t)\left(B_{t+\Delta}-B_{t}\right)$ expresses the random component of the motion due to random collisions. More specifically the collisions increased with the temperature of the fluid; the influence of temperature is modeled by the diffusion coefficient $\sigma(x, t)$. We note that the model (13) implies that, due to the normality of the Brownian increment, the displacement of the particle $X_{t+\Delta}-x$ is approximated by a Gaussian random variable of mean $\mu(x, t) \Delta$ depending on the drift and of variance $\sigma(x, t) \sqrt{\Delta}$ depending on the diffusion coefficient.

Heuristically, an 'SDE' is obtained from Equation (13) by replacing $\Delta$ by $d t,\left(B_{t+\Delta}-B_{t}\right)$ by $d B_{t}$ and $X_{t}+\Delta-X_{t}$ by $d X_{t}$. Then we have the following definition.

Definition 2.1. Let $\left(B_{t}\right)$ be a d-dimensional Brownian motion. Let $\mu: \mathbb{R}^{+} \times \mathbb{R}^{d} \rightarrow \mathbb{R}^{d}$ and $\sigma(x, t): \mathbb{R}^{+} \times \mathbb{R}^{d} \rightarrow \mathcal{M}^{d}$ be given functions $\left(\mathcal{M}^{d}\right.$ denoting the set of square matrix of size $\left.d\right)$. An $\mathrm{SDE}$ is defined as

$$
d X_{t}=\mu\left(X_{t}, t\right) d t+\sigma\left(X_{t}, t\right) d B_{t},
$$

where $\left(X_{t}\right)$ is the unknown process. The function $\mu$ is referred to as the drift while the function $\sigma$ is called the diffusion coefficient.

\section{Solution of SDE}

There are two types of solutions respectively called 'strong' and 'weak solutions'. A strong solution is a weak solution but the reverse is false.

Definition 2.2. Let $\mathcal{F}_{t}$ the field induced by the initial condition $X_{0}$ and the Brownian motion $\left(B_{t}\right)$ which drives the stochastic differential (14). We say that Equation (14) has a strong solution $\left(X_{t}\right)$ on the probability space $(\Omega, \mathcal{F}, P)$ with respect to $\left(B_{t}\right)$ and initial condition $X_{0}$ if the stochastic process $X_{t}$ satisfies (14), has continuous paths and that $X_{t}$ is $\mathcal{F}_{t}$-measurable for all $t$.

The fact that $X_{t}$ is $\mathcal{F}_{t}$-measurable is crucial. It means that $X_{t}$ depends only on the historic of the Brownian motion which drives the SDE and the initial condition. Then we can interpret $X_{t}$ as an output of the system parametrized by $\mu(x, t)$ and $\sigma(x, t)$ whose input is the Brownian motion $\left(B_{t}\right)$. It reflects the principle of causality of the system. If $X_{t}$ could depend on the future, that is on $B_{s}$ with $s>t$, causality would fail.

The concept of strong solution relies on the fact that the Brownian motion is given. A weak solution of an SDE consists in building at the same time a couple of processes $\left(X_{t}, B_{t}\right)$ where $\left(X_{t}\right)$ is a solution of the SDE driven by the Brownian $\left(B_{t}\right)$. We will not give the exact definition of weak solution as it has technical points not of interest for the understanding of the concept.

Then the solution of the SDE is written as

$$
X_{t}=X_{0}+\int_{0}^{t} \mu\left(X_{s}, s\right) d s+\int_{0}^{t} \sigma\left(X_{s}, s\right) d B_{s} .
$$

We note that the fact that the two integrals are defined is equivalent to the fact that $X_{t}$ is (strong or weak) solution. In particular the integral with integrand $\mathrm{dB}_{\mathrm{t}}$ is a random variable $\mathcal{F}_{\mathrm{t}}$-measurable. Details of the construction of such integrals are given in [20, Chapter 4].

\section{Fractional Brownian motion}

$\mathrm{fBm}$ was introduced to model scale-invariant phenomenons processes showing long-range dependence. Kolmogorov [23] developed a turbulence theory based on two hypotheses of scale invariance. In his study of long-term storage capacity and design 
of reservoirs, Hurst [24] observed hydrological events invariant to changes in scale. Mandelbrot and Van Ness [21] defined the fBM of exponent has a 'moving average of $d B(t)$, in which past increments of $B(t)$ are weighted by the kernel $(t-s)^{2 \mathfrak{h - 1}}$.' This kernel is at the origin of the long-range dependence property (for a certain choice of parameter $\mathfrak{h}$ ). The parameter $\mathfrak{h}$ is known as the Hurst index or Hurst parameter. In this section, we define $\mathrm{fBm}$ and give its main properties. $\mathrm{FBm}$ is then defined in dimension $d$.

\section{Self-similarity and $\mathrm{fBm}$}

A real-valued stochastic process $\left(X_{t}\right)$ is 'self-similar' with index $\mathfrak{h}>0(\mathfrak{h}-s s)$ if, for any $a>0$ the processes $\left(X_{a t}\right)$ and $\left(a^{\mathfrak{h}} X_{t}\right)$ have the same finite dimensional distributions. Then a Gaussian $\mathfrak{h}-$ ss process $\left(B_{t}^{\mathfrak{h}}\right)$ with stationary increments and Hurst index $0<\mathfrak{h}<1$ is an ' $\mathrm{fBm}$ '.

Now we give some properties of the $f B m$. First, the $f B m$ has continuous paths. We have $\mathbb{E}\left(B_{t}^{\mathfrak{h}}\right)=0$ for all t. It is said to be standard if the variance of $B_{1}^{\mathfrak{h}}$ is equal to one. For the standard $\mathrm{fBm}$ we have

$$
\operatorname{Cov}\left(B_{t}^{\mathfrak{h}}, B_{s}^{\mathfrak{h}}\right)=\frac{1}{2}\left(|t|^{2 \mathfrak{h}}+|s|^{2 \mathfrak{h}}-|t-s|^{2 \mathfrak{h}}\right)
$$

Then we can show that an $\mathrm{fBm}$ with $\mathfrak{h}=1 / 2$ is simply a (onedimensional) Brownian motion.

\section{Long-range dependence}

A stationary time series $\left(X_{n}\right)_{n \in \mathbb{N}}$ exhibits 'long-range dependence' if $\operatorname{Cov}\left(X_{n}, X_{0}\right) \rightarrow 0$ as $n \rightarrow \infty$ but

$$
\sum_{n=0}^{\infty}\left|\operatorname{Cov}\left(X_{n}, X_{0}\right)\right|=\infty .
$$

In other words the covariance between $X_{0}$ and $X_{n}$ tends to 0 but so slowly that their sum diverges. Then we define the stationary process known as fractional Gaussian noise

$$
X_{k}=B_{k+1}^{\mathfrak{h}}-B_{k}^{\mathfrak{h}}, \quad k \in \mathbb{N}
$$

where $\left(B_{t}^{\mathfrak{h}}\right)$ is a standard $\mathrm{fBm}$ of Hurst index $\mathfrak{h}$. Due to the properties of $\mathrm{fBm}$ the fractional Gaussian noise $\left(X_{n}\right)$ is a stationary-centered Gaussian process with auto-covariance function

$$
\gamma(k)=\mathbb{E}\left(X_{i+k} X_{i}\right)=\frac{1}{2}\left(|k+1|^{2 \mathfrak{h}}+|k-1|^{2 \mathfrak{h}}-2|k|^{2 \mathfrak{h}}\right) .
$$

Then for $k \neq 0$ we can show that $\gamma(k)=0$ if $\mathfrak{h}=1 / 2, \gamma(k)<0$ if $0<\mathfrak{h}<1 / 2$ and $\gamma(k)>0$ if $1 / 2<\mathfrak{h}<1$. Now, for $\mathfrak{h}=1 / 2$ we have

$$
\gamma(k)=\mathfrak{h}(2 \mathfrak{h}-1)|k|^{2 \mathfrak{h}-1}+o(1),
$$

where $o(1) \rightarrow 0$ as $k \rightarrow \infty$. Consequently $\gamma(k) \rightarrow 0$ as $k \rightarrow \infty$ for $0<\mathfrak{h}<1$. From Equation (20) we deduce

$$
\begin{aligned}
& \sum_{k=0}^{\infty} \gamma(k)=\infty, 1 / 2<\mathfrak{h}<1, \\
& \sum_{k=0}^{\infty} \gamma(k)<\infty, 0<\mathfrak{h}<1 / 2 .
\end{aligned}
$$

Consequently, if $1 / 2<\mathfrak{h}<1$, fractional Gaussian noise (hence $\mathrm{fBm})\left(\mathrm{X}_{n}\right)$ exhibits long-range dependence.

\section{Stochastic integration and $\mathrm{fBm}$}

As stated in the introduction, [21] define the $\mathrm{fBm}$ as a moving average of $d B_{t}$. Decreusefond and Ustünel [25] shows that $\mathrm{fBm}$ can be written as the following stochastic integral driven by Brownian motion:

$$
B_{t}^{\mathfrak{h}}=\int_{0}^{t} K_{\mathfrak{h}}(t, s) d B_{s},
$$

where the properties and analytical form of function $K_{\mathfrak{h}}(t, s)$ (called kernel) are given in [25].

\section{Multivariate $\mathrm{fBm}$}

Coutin and Quian [26] give the following definition of a $d$ dimensional fBm.

Definition 2.3. An $\mathrm{fBm}$ in dimension $d>1$ is the random vector $B_{t}^{\mathfrak{h}}=\left(B_{t}^{\mathfrak{h}, 1}, \ldots, B_{t}^{\mathfrak{h}, d}\right)$ where all coordinates $B_{t}^{\mathfrak{h}, i}$ are independent one-dimensional fractional Brownian motions of Hurst parameter $0<\mathfrak{h}<1$.

Again a d-dimensional $\mathrm{fBm}$ reduces to a d-dimensional Brownian motion in the case $\mathfrak{h}=1 / 2$.

\section{SDE driven by $\mathrm{fBm}$}

We can extend the SDE (14) to define a ( $d$ - dimensional) stochastic differential driven by a (d-dimensional) $\mathrm{fBm}$ of Hurst index $0<\mathfrak{h}<1$

$$
d X_{t}=\mu\left(X_{t}, t\right) d t+\sigma\left(X_{t}, t\right) d B_{t}^{\mathfrak{h}} .
$$

The same concepts of strong and weak solutions hold for the SDE (22). The SDE driven by Brownian motion (14) is of the form of the SDE (22) with $\mathfrak{h}=1 / 2$.

In the remainder of this paper, we will call diffusion any processes solution of (22). We note that it does not match with the definition of [19, Chapter 15, Section 1] given in Diffusion Process section, as the Markov property no longer holds due to the correlations between the fBm increments.

\section{Summary}

In this section, we presented Brownian motion from a probabilistic perspective. This process is of paramount importance in mathematics, physics and biophysics. We also presented diffusion as solutions of an SDE and introduced the $\mathrm{fBm}$ which generalized Brownian motion adding correlations between its increments. 
In the next section, we give the physical derivation of Brownian motion. We will also describe the motion models used in biophysics for describing intracellular dynamics, with a particular emphasis on the diffusion models defined in this section.

\section{Diffusion for modeling intracellular trajectories}

First, we present the physical models underlying Brownian motion. More specifically, we introduce the theory of [27] and the Langevin approach. Then, we present subdiffusion processes which is often split in two parts: anomalous and confined diffusion. Finally, we deal with superdiffusion. Also, we compute the MSD for each presented model as it is the criterion to classify the motion model as free diffusion, subdiffusion or superdiffusion. We note that we also exhibit motion models which are not diffusion in the sense of Stochastic Processes, Brownian motion, and Diffusions section, in particular in the case of subdiffusions. Another general presentation of theoretical transport models can be also found in $[28]$.

\section{Einstein's approach}

In this section, we present the approach of [27] introduced for modeling the motion of 'small suspended particles' in a liquid. We develop the concept of Brownian motion in the exact same way as [27]. First, we depict the related physical experiment. Secondly, we show that the concentration of suspended particles is governed by a diffusion in the sense of Fick. Finally, the motion of individual suspended particles is modeled by a process corresponding to Brownian motion.

\section{Physical context}

Einstein considers a particular physical situation. In 1st place, he assumes that $\mathrm{z}$ moles of a chemical species are dissolved in a liquid of volume $\mathrm{V}$. He also supposes that the solute is confined in a volume $V^{\star}$ separated from the pure solvent by a wall that is permeable to the solvent but not to the solute. In this situation, the solute produces a pressure on the wall called the osmotic pressure. Provided $z / V^{\star}$ is small enough, that is the solute concentration is low, we have

$$
p V^{\star}=R T z
$$

where $p$ is the osmotic pressure, $R$ is the gas constant and $\mathrm{T}$ is the temperature. Secondly, instead of the solute, Einstein considers suspended particles. Now the wall is permeable to the solvent but not to the particles. In this case, the theory of thermodynamics does not expect that the suspended particles will produce an osmotic pressure on the wall. However, according to the molecular-kinetic of heat, the only difference between a dissolved molecule and a suspended body is their size. Then, Einstein points out that both the dissolved molecules and the suspended particles should produce the same osmotic pressure as long as their number is equal. Then he assumes that 'the suspended bodies perform an irregular, albeit very slow, motion in the liquid due to the liquids molecular motion'. This motionwe will see later that it corresponds to Brownian motion-is at the origin of the osmotic pressure. In fact, when the moving particles bounce on the wall, they exert a pressure as in the case of the solute. Then we can derive a similar equation as (23)

$$
p V^{\star}=R T \frac{n}{N}
$$

where $n$ is the number of suspended particles and $N$ the Avogadro number. Then $n / N$ is the number of moles of the suspended particles.

In [27], Einstein focused on the motion of particles subjected to forces and moving along a single direction.

\section{Fick's diffusion}

In this paragraph, we are interested in the evolution of the concentration in space and time $v(x, t)=n(x, t) / d x$, where $n(x, t)$ in the number of suspended particles at time $t$ in the small volume $d x$. Einstein [27] assumes that a force $K$, depending on the position but not on the time, acts on each particle.

First, at the equilibrium we have

$$
K v-\frac{\partial p}{\partial x}=0
$$

that is the force $\mathrm{K}$ and the force induced by the pressure $p$ compensate each other. Using the definition of $v$ and Equation (24), we can rewrite Equation (25) as

$$
K v-\frac{R T}{N} \frac{\partial v}{\partial x}=0
$$

On the other hand, the concentration $v$ is governed by a diffusion in the sense of [29]. In this case, diffusion refers to the evolution of a macroscopic quantity as the heat in a metal or the concentration of a chemical species in a liquid. It is characterized by the two laws of [29]. Once combined, they give the diffusion equation which is written in our case as

$$
\frac{\partial v}{\partial t}=D \frac{\partial^{2} v}{\partial^{2} x}
$$

where $D$ is the diffusion coefficient characterizing the diffusion.

Now, to fully determine the diffusion of $v$ we need to derive $D$ as a function of the parameters of the problem. To this end, we use the 1st law of [29] stating that 'the diffusion flux between two points of different concentrations in the fluid is proportional to the concentration gradient between these points'. In our case it can be written as

$$
J=-D \frac{\partial v}{\partial x}
$$

where $J$ is the diffusion flux and $D$ is the diffusion coefficient characterizing the diffusion. Now we must derive the diffusion flux $J$ that is the number of particles going through an area of unit one per unit of time. Einstein [27] assumes that the suspended particles are spheric of radius a. Additionally, if the liquid has coefficient of viscosity $k$, then the force $K$ gives to each particle the velocity,

$$
\frac{v K}{6 \pi k a}
$$




\begin{tabular}{|c|c|c|c|}
\hline \multicolumn{2}{|c|}{$\nu\left(x-\Delta_{x}, t\right) d x$} & \multicolumn{2}{|c|}{$\nu(x, t+\Delta) d x$} \\
\hline$x-\Delta_{x}$ & $x-\Delta_{x}^{\prime}+d x$ & $x$ & $x+d x$ \\
\hline
\end{tabular}

Figure 5. Scheme illustrating the transfer of particles from $x-\Delta_{x}$ to $x$ between the times $t$ and $t+\Delta$. There are $v(x-\Delta x, t) d x$ particles in $[x-\Delta x, x-\Delta x+d x]$ at time $t$. Among them a proportion of $\phi(\Delta x) d \Delta x$ jumps to $[x, x+d x]$ between $t$ and $t+\Delta$. Integrating over all the displacements $\Delta x$, we obtain $v(x, t+\Delta) d x$ particles at time $t$ in $[x, x+d x]$.

Consequently the diffusion flux is

$$
J=\frac{v K}{6 \pi k a} .
$$

In fact, a dimension analysis reveals that the inverse of a volume $\left(v=n / V^{\star}\right)$ multiplied by a velocity (Equation (29)) defines a flux.

Finally, the 1st law of [29] gives

$$
\frac{v K}{6 \pi k a}=-D \frac{\partial v}{\partial x}
$$

From Equations (26) and (31), the Fick's diffusion governing $v$ has for diffusion coefficient

$$
D=\frac{R T}{N 6 \pi k a} .
$$

\section{Brownian motion}

Finally, Einstein [27] models the 'disordered motions' due to thermal molecular agitation of the $n$ suspended particles. More importantly, Einstein links these individual motions to the Fick's diffusion examined in the previous paragraph. He assumes that the motions of individual particles are independent from each other. Moreover, he assumes that the displacements of a same particle on consecutive time intervals are independent as long as these time intervals are not too small. Then in the following, we denote $\Delta$ the length of the time interval which is small compared to the observable time intervals but still satisfy the independence property of displacements. We recall that the displacements occur along the $x$-axis only. We denote $\Delta_{x}$ the displacement occurring during the period $\Delta$. Einstein [27] assumes that $\Delta_{x}$ is a random variable whose distribution function $\phi$ is symmetric. Then the probability that a particle experiences a displacement lying between $u$ and $u+d u$ is $\phi(u) d u$. The average number of particles experiencing such a displacement during a period $\Delta$ is

$$
d n=n \phi(u) d u .
$$

Now, we can deduce the number of particles $v(x, t+\Delta) d x$ from the the numbers of particles at time $t$ and $\phi$. In Figure 5, we show how the particles go from $x-\Delta_{x}$ at time $t$ to $x$ at time $t+\Delta$ using Equation (33). Integrating over all the possible displacements we get

$$
v(x, t+\Delta) d x=d x . \int_{\mathbb{R}} v\left(x-\Delta_{x}, t\right) \phi\left(\Delta_{x}\right) d \Delta_{x} .
$$

As $\Delta$ is small we can expand $v(x, t+\Delta)$ as

$$
v(x, t+\Delta)=v(x, t)+\Delta \frac{\partial v(x, t)}{\partial t} .
$$

We also expand the left side of Equation (34) in Taylor series

$$
\begin{aligned}
\int_{\mathbb{R}} v\left(x-\Delta_{x}, t\right) \phi\left(\Delta_{x}\right) \mathrm{d} \Delta_{x}= & v(x, t) \times 1+\frac{\partial v(x, t)}{\partial x} \times 0 \\
& +\frac{\partial^{2} v(x, t)}{\partial^{2} x} \int_{\mathbb{R}} \frac{\Delta_{x}^{2}}{2} \phi\left(\Delta_{x}\right) \mathrm{d} \Delta_{x},
\end{aligned}
$$

where we use that $\int \phi(u) d u=1$ as $\phi$ is a distribution function and $\int u \phi(u) d u=0$ as $\phi$ is symmetric. We can equalize the right side of the two previous equations according to the equality given in Equation (34). Then, we deduce that $v$ respects the diffusion equation (27) predicted by the theory in [29] with diffusion coefficient given by

$$
D=\frac{1}{\Delta} \int_{\mathbb{R}} \frac{\Delta_{x}^{2}}{2} \phi\left(\Delta_{x}\right) \mathrm{d} \Delta_{x}
$$

Therefore, with a specific definition of the individual motion of $n$ independent particles, Einstein [27] shows that the concentration of such particles follows the Fick's equation.

At this step, he [27] shows only assumed that the displacement of each particle over consecutive time intervals-for intervals not too small-are independent random variables from a symmetric distribution $\phi$. Consequently, the particle motion fulfills the independence property of the Brownian increment, see Brownian motion section. For the moment, we cannot see why the displacement of the particles should be Gaussian as for Brownian particle. This link can be made by solving the diffusion equation (27).

We need additional conditions to solve Equation (27). Until this point, we have used the same coordinate system for all the particles. As all the particles are independent, we can define one coordinate system for each particle. Einsitein [27] states that the center of gravity of each particle at time $t=0$ is the origin of their coordinate system. Then $v(x, t) d x$ now denotes the number of particles whose displacements between the times 0 and $t$ are comprised between $x$ and $x+d x$. In other words, $x$ denotes the displacement and not the absolute position in a common coordinate system anymore. Function $v$ still verifies Equation (27) under this new scheme. Now we have the straightforward conditions

$$
\begin{aligned}
v(x, 0) & =0, \quad \forall x \neq 0 \\
\int_{\mathbb{R}} v(x, 0) d x & =n .
\end{aligned}
$$

Finally, the solution of the diffusion equation (27) with conditions (36) is

$$
v(x, t)=n \frac{e^{\frac{-x^{2}}{4 t \mathrm{t}}}}{\sqrt{4 \pi \mathrm{Dt}}},
$$

where $x$ is interpreted as a displacement. With this meaning of $x, e^{-x^{2} /(4 \mathrm{Dt})} / \sqrt{4 \pi \mathrm{Dt} d x}$ is the probability that the displacement of a single particle lies in $[x, x+d x]$. Therefore, the particle displacement is Gaussian. We also know that the displacements over consecutive time intervals are independent. Then the motion of the suspended particles defined by [27] corresponds to the Brownian motion defined in Brownian motion section. Therefore, the physical derivation of Brownian motion by [27] is equivalent to the so-called Wiener process in mathematics. Due to the physical constraints, the diffusion coefficient $D$ has a particular value given by Equation (32). 
We can extend this theory to the $d$-dimensional case ( $d=$ $2,3)$. In this context, each component follows a one-dimensional Brownian motion and the components are independent from each other. Not surprisingly, it corresponds to the Definition 2.3 of multi-dimensional Brownian motion.

Remark 3.1. We note that, in this paper, in case of the onedimensional Brownian motion $\left(B_{t}\right)$ the diffusion coefficient $\sigma$ is defined as $\sigma=\operatorname{Var}\left(B_{1}\right)$. Then we have the relationship $\sigma=2 \mathrm{D}$.

Remark 3.2. From Equations (33) and (37) and the definition of $\phi$ we deduce that $\phi(x)=e^{-x^{2} /(4 D \Delta)} / \sqrt{4 \pi D \Delta}$. It is coherent with the equality (35).

\section{Langevin's approach}

Physicists define the motion of suspended particles in another way using the approach of [30] (see [31] and [32, Chapter 1]). This motion is sometimes referred to as Brownian motion which can be confusing. In this subsection, we present this alternative approach. First, we introduce the underlying physical model and the corresponding hypotheses about the particle motion. Secondly, we show that, in this case, the particle movement is governed by a well-known SDE. Thirdly, we explain why the particle motion defined by Einstein [27] and by Langevin [30] is mixed up. Finally, we explain which concept of Brownian motion we will use in the paper. In this subsection, we derive the model directly in dimension $d$.

\section{Langevin quation}

Langevin [30] characterizes the particle motion through the $d$ dimensional (Langevin) equation

$$
m \frac{d v(t)}{d t}=-\zeta v(t)+L(t)
$$

where $v: \mathbb{R}^{+} \mapsto \mathbb{R}^{d}$ is the velocity of the particle, $m$ its mass, $\zeta>0$ the friction coefficient and $L: \mathbb{R}^{+} \mapsto \mathbb{R}^{d}$ a random force resulting from the collisions with the surrounding particles. In case of spherical particles of radius a immersed in a liquid of viscosity coefficient $k$, the friction coefficient is $\zeta=6 \pi k a$ where $k$ is the viscosity coefficient of the surrounding liquid.

Uhlenbeck and Ornstein [33] constrained L(t) with two additional assumptions. First, the mean of $L(t)$ over a large number of independent colliding particles is 0 , that is $\mathbb{E}(L(t))=0_{d}$, where $0_{d}$ is the null vector of $\mathbb{R}_{d}$. In their physical model, [33] also assume that the colliding particles are similar to the particle of interest and have same initial speed $v_{0}$. Secondly, the autocorrelation function is given by

$$
\mathbb{E}\left(\mathrm{L}(\mathrm{t}) \mathrm{L}(\mathrm{s})^{\mathrm{T}}\right)=\sigma \delta(t-\mathrm{s}) \mathbf{I}_{\mathrm{d}},
$$

where $\sigma>0$ is a constant, $\delta$ is the Kronecker function and $I_{d}$ the identity matrix of size $d$. The idea is that each collision is practically instantaneous and that successive collisions are uncorrelated. Actually, Uhlenbeck and Ornstein [33] originally model the autocorrelation function as a function of $t-s$ with a sharp peak of width equal to the duration of a single collision. The autocorrelation (39) is preferred nowadays [34, Chapter 9]. Such a force $L(t)$ is called a Langevin force.

\section{Ornstein-Uhlenbeck process}

We did not fully define the stochastic process $L(t)$ as we provide only information on its 1st and 2nd moment. Such a process is known as white noise in statistics. If we further assume that $L(t)$ is Gaussian, we entirely define this process as a Gaussian process is determined by its 1st two moments. Then $L(t)$ is called a Gaussian white noise. As explained in [19, Chapter 15, Subsection 14], the Gaussian white noise L(t) can be informally defined as the derivative of the Wiener process-equivalently the mathematical Brownian motion defined in Section 2.2 $L(t)=\sigma d B_{t} / d t$. We use the word informally as in fact the Wiener process is nowhere differentiable. Finally, we can rewrite the Langevin equation (38) as the $d$-dimensional SDE

$$
m d v(t)=-\zeta v(t) d t+\sigma d B_{t} .
$$

The solution of the stochastic equation (40) is known as the Ornstein-Uhlenbeck process. It is a Gaussian process with

$$
\begin{gathered}
\mathbb{E}(U(t))=0_{d}, \\
\mathbb{E}\left(\mathcal{U}(t) \cup(s)^{T}\right)=\frac{\sigma^{2}}{2 \zeta m} e^{-(\zeta / m)|t-s|} \mathbf{I}_{d} .
\end{gathered}
$$

Waterston and Rayleigh [35] states that, at the equilibrium (that is as $t \rightarrow \infty$ ), the mean square velocity verifies

$$
\lim _{t \rightarrow \infty} \mathbb{E}\left(\|v(t)\|_{2}^{2}\right)=d \frac{k_{B} T}{m},
$$

where $k_{B}$ is the Boltzmann constant and $T$ is the temperature. Each component of the velocity vector has the same variance, so that

$$
\lim _{t \rightarrow \infty} \mathbb{E}\left(v_{i}(t)^{2}\right)=\frac{k_{B} T}{m}, \quad i=1, \ldots, d .
$$

Then equalizing the variances of $v_{i}(t)$ obtained with Equation (42) with $t=s$ and obtained with Equation (44), we have the relationship

$$
\sigma=\sqrt{2 \zeta k_{B} T}
$$

Finally, the Brownian motion of [30] is defined as

$$
X_{t}=\int_{0}^{t} v(s) d s,
$$

where $v(t)$ is the Ornstein-Uhlenbeck process solution of the SDE (40). Due to the Gaussian nature of $v(t),\left(X_{t}\right)$ is also a Gaussian process.

\section{Mean square displacement}

One reason explaining the confusion between the particle motion respectively defined in [27] and [30] is that they both exhibit a linear MSD asymptotically. In the case of the 
d-dimensional Brownian motion of [27], we can easily show that the MSD is

$$
\begin{aligned}
\mathbb{E}\left(\left\|X_{t}-X_{0}\right\|^{2}\right) & =d 2 D t \\
& =d \frac{2 R T}{N 6 \pi k a} t \\
& =d \frac{2 k_{B} T}{\zeta} t
\end{aligned}
$$

where $k_{B}=R / N$ is the Boltzmann constant and $\zeta=6 \pi k a$ is the friction coefficient.

In the case of the motion defined by [30] (assuming $X_{0}=0$ for simplicity) we have

$$
\begin{aligned}
\mathbb{E}\left(\|\| X_{t}-X_{0}\|\|^{2}\right) & =\sum_{i=1}^{d} \mathbb{E}\left(\int_{0}^{t} \int_{0}^{t} v^{i}(s) v^{i}(u) d s d u\right) \\
& =d \int_{0}^{t} \int_{0}^{t} \mathbb{E}\left(v^{1}(s) v^{1}(u)\right) d s d u \\
& =d \frac{2 k_{B} T}{\zeta}\left(t-\frac{m}{\zeta}\left(1-e^{-(\zeta / m) t}\right)\right) \\
& =d \frac{2 k_{B} T}{\zeta} t+o(t),
\end{aligned}
$$

where $o(t) \rightarrow 0$ as $t \rightarrow \infty$.

\section{Choice of the definition of Brownian motion}

Each approach relies on different physical models. We emphasize that the Brownian motion in [27] (corresponding to the Wiener process) is nowhere differentiable and then has a rough (but still continuous) path. On the other hand, the particle motion defined in [30] is differentiable due to its definition as the integration of the Ornstein-Uhlenbeck process (Equation (46)). Then its path is smooth. Bressloff [36] argues that both processes can be used to model intracellular dynamics in the case where the particle evolves freely inside the cytosol or along the plasma membrane.

In what follows, Brownian motion will refer to the motion defined by Einstein [27]. It corresponds to the mathematical Brownian motion defined in Brownian motion section called also Wiener process in the mathematical literature.

\section{Subdiffusion}

Subdiffusion, which includes confined diffusion and anomalous diffusion, is the translations of several biological scenarios [28, 37]. In this subsection, we present models associated with these two types of diffusion. We note that certain models are called diffusion even if they are not solutions of the SDE.

\section{Anomalous diffusion}

In biophysics, $[2,18]$, an 'anomalous diffusion' $\left(X_{t}\right)$ is characterized by an MSD which is proportional to the monome $t^{\beta}$,

$$
\mathbb{E}\left(\left\|X_{t}-X_{0}\right\|^{2}\right) \propto t^{\beta},
$$

with $\beta<1$. The 1st two presented models are solutions of an SDE driven by $\mathrm{fBm}(22)$ (the first being simply $\mathrm{fBm}$ ). Then we present other types of processes used in biophysics, also reported in details in [37].

Fractional Brownian motion. As a particle moves through the cytoplasm, the latter pushes it back, due to macromolecular crowding and the presence of elastic elements generating correlations in the particles trajectory [38]. An fBm with Hurst index $0<\mathfrak{h}<1 / 2$ is a good candidate to model this situation. First, it is straightforward to show that its MSD is given by (49) with $\beta=2 \mathfrak{h}<1$ (see Equation (16)). Secondly, we saw in Fractional brownian motion section that $\mathrm{fBm}$ has its increments negatively correlated when $0<\mathfrak{h}<$ 1/2. As an example, [39] study the mechanisms underlying subdiffusive motion in live Escherichia coli cells thanks to fluorescently labeled chromosomal loci and RNA-protein particles. They conclude that the observed motion was well modeled by $\mathrm{fBm}$.

Generalized Langevin equation (GLE). As we have just explained, particles can be slowed by the contrary current due to the viscoelastic properties of the cytoplasm (see also [9] for details). This time we are interested in long-time correlations (and not just correlations) in diffusive motion. Then Kou [31] models such phenomenon with SDEs driven by the $\mathrm{fBm}$ with Hurst index $1 / 2<\mathfrak{h}<1$; in fact we saw in Fractional brownian motion section that in this case $\mathrm{fBm}$ exhibits long-range dependence. Then Zwanzig [40] and Chandler [41] proposed the GLE

$$
m \frac{d v(t)}{d t}=-\zeta \int_{-\infty}^{t} v(u) K(t-u) d u+G(t)
$$

where, in comparison with the Langevin equation (38), $G(t)$ is a noise having memory replacing the memoryless white noise $L(t)$; the velocity is convolved with a kernel $K$. These two features make the solution of the Equation (50) a non-Markovian process. Analogically to $v(t)$ in Equation (43), both $K$ and $G$ must fulfill a physical constraint called the fluctuation-dissipation principle in [41]

$$
\mathbb{E}\left(G(t) G(s)^{T}\right)=2 \zeta k_{B} T K(t-s) I_{d} .
$$

Not surprisingly, we observe that if we choose $K=\delta$-the Dirac function-we find that the GLE (50) is equivalent to the Langevin equation (38) and the condition on the 2nd moment (51) is equivalent to the condition (39). Kou [31] chooses to define $G(t)$ as fractional Gaussian noise (18) with Hurst index $1 / 2<\mathfrak{h}<1$ for exhibiting long-range dependence. From condition (51), they deduce the kernel $K$ (noted now $K_{\mathfrak{h}}$ )

$$
K_{\mathfrak{h}}(t)=\left.2 \mathfrak{h}(2 \mathfrak{h}-1)|t|\right|^{2 \mathfrak{h}-2} .
$$

Then the related SDE is

$$
m d v(t)=-\zeta\left(\int_{-\infty}^{t} v(u) K(t-u) d u\right) d t+\sigma d B_{t}^{\mathfrak{h}}
$$

where $\sigma=2 \zeta k_{B} T$ and $\left(B_{t}^{\mathfrak{h}}\right)$ is an fBm with $1 / 2<\mathfrak{h}<1$. Finally, Kou [31] shows that the integrated process $X_{t}=\int v(u) d u$ verifies as $t \rightarrow \infty$

$$
\mathbb{E}\left(\left\|X_{t}-X_{0}\right\|^{2}\right) \propto t^{2-2 \mathfrak{h}} .
$$


It fulfills the MSD condition (49) asymptotically with $\beta=2-2 \mathfrak{h}<1$ for $1 / 2<\mathfrak{h}<1$.

Continuous time random walk (CTRW). Intracellular particles can also bind to molecular complexes. Then the particle motion is a permanent switch between binding events and movement toward another spot where it can bind again. Scher and Montroll [42] introduce the CTRW to model anomalous transport properties of charge carriers in amorphous materials. In their framework, the electron dynamics are successively trapped in different energy wells; the total time spent in the trapped states is much larger than the time spent in free motion.

CTRW is a pure-jump stochastic process and formally it cannot be considered as a diffusion process as defined in the paper (since it is not continuous). Nevertheless, as CTRW generalizes random walks, it is considered as a process leading to anomalous diffusion. In this model, a particle performs random jumps whose step length is generated by a probability density with finite 2nd moments. The waiting times between jumps are assumed to be distributed according to a probability distribution $\psi(t)$. If $\psi(t)$ has a finite 1st moment that is $\int t \psi(t) d t<\infty$ then the MSD of the CTRW is linear in time. For instance, we can use the exponential distribution

$$
\psi(t)=(1 / \tau) e^{-t / \tau}, t>0,
$$

where $\tau>0$ is called the characteristic time. We note that, in this case, the random walk has the Markov property (due to the memoryless property of the exponential distribution). On the contrary, if $\int t \psi(t) d t=\infty$ the MSD of the CTRW is given by (49). A typical choice is a power law distribution

$$
\psi(t)=1 /(1+t / \tau)^{1+\beta}, t>0
$$

with $\tau>0$ the characteristic time and $0<\beta<1$.

In neurobiology, Zhizhina et al. [43] propose to investigate CTRW to model the axon growth. The growth of an axon to its target is guided by chemical signals from the cellular environment. The authors describe this interaction by a random waiting time thereby defining a CTRW. They observe that 'normal' axons and 'mutant' axons are driven by CTRW with different waiting time distribution.

Random walk on fractal. The inner environment of a cell is crowded with small solutes and macromolecules which occupy $10-50 \%$ of the volume [45]. If the concentration of obstacles is sufficiently high, the MSD of the particle is given by Equation (49) $[7,44]$. In that case, the MSD is ill-defined as Brownian diffusion occurs locally, but the MSD exponent $\beta$ is $<1$ at larger time scale, depending on obstacle density and size compared to the particle (see [46]).

Since the domain has a fractal-like structure, a popular model to represent the underlying dynamics of particles is to consider random walks on percolation clusters [44]. Here we present the model on a 2-dimension square lattice. Each vertex of the lattice has probability $1-p$ to be an obstacle, that is the particle cannot go on this kind of vertex. The other vertices can be occupied by particles. They form connected clusters on which particles are assumed to undergo a random walk. In this particular case, there exists a critical probability $p_{c}=0.592745$ below which there exist only finite clusters, and above which there exists one infinite cluster (see Figure 10 in [44]). When $p=p_{c}$, the random walk on the infinite cluster has its MSD given by Equation (49) [44]. In the literature of diffusion on fractals, it is established that the MSD (49) is a function of $\beta=d / d_{w}$ where $d$ is the dimension and $d_{w}$ is related to the fractional dimension of the random walk. If $d=2$, the fractional dimension of the random walk on a square lattice with $p=p_{c}$ is $d_{w}=2.8784$ [47] leading to $\beta=0.6948$. Havlin and Ben-Avraham [44] also consider other choices of $p$ and random walks on both the finite and infinite percolation clusters. However, in these cases, the MSD is not a power function. As another two-dimensional example, the fractional dimension of a random walk on the Sierpinski gasket fractal gives $d_{w}=2.32$ (then $\beta=0.8621$ ) [44]. Berry and Chaté [8] argue that the exponents $\beta$ observed from real experiments span a wide range of values and that random walks on fractal cannot model all these possibilities. Then several authors [7, 8] prefer relying on Monte Carlo simulations with different designs of obstacles (mobiles or not) to propose a model explaining the observed power function form of the MSD.

\section{Confined diffusion}

The MSD of a confined diffusion is not a simple power law of time as generally assumed for other diffusion processes. Actually, in biophysics $[2,17]$, a 'confined diffusion' $\left(X_{t}\right)$ is characterized by a MSD of the form

$$
\mathbb{E}\left(\left\|X_{t}-X_{0}\right\|^{2}\right)=\frac{r_{c}^{2}}{a}\left(1-b e^{-c \sigma^{2} t /\left(2 r_{c}^{2}\right)}\right),
$$

where parameter $r_{c}$ is the characteristic size of the region of confinement, $a$ is a scale parameter and $b$ and $c$ depends on the shape of the region. Parameter $\sigma>0$ is the constant diffusion coefficient. We present two models of confined diffusion and give their MSDs. For the 1st model, the MSD (57) is a simplification of the true MSD. Here we find the MSD (57) for a particular case of the 2nd model. We note that parameter a does not appear in $[2,17]$. We use this extra scale parameter $a$ to have the common expression (57) for the MSD of the two presented models.

Diffusion within confined geometries. The plasma membrane is parceled up into compartments where proteins undergo shortterm confined diffusion. More specifically these compartments are separated by the actin-based membrane skeleton [48]. Then the motion can be modeled by the SDE (14) adding boundary conditions. Equation (57) is based on the 1st term of the exact series solution of the MSD of a Brownian particle trapped in a square or circular corral (in dimension 2) or in a sphere (in dimension 3) $[49,50]$. As an example, Bickel [51] shows that, for a certain type of boundary condition, the MSD of a Brownian motion confined in a circular domain of radius $r_{c}$ is given by

$$
\mathbb{E}\left(\left\|X_{t}-X_{0}\right\|^{2}\right)=r_{c}^{2}\left(1-8 \sum_{i=1}^{\infty} \exp \left[-\iota_{1 i}^{2} \frac{t}{\tau}\right] \frac{1}{\iota_{1 i}^{2}\left(\iota_{1 i}^{2}-1\right)}\right),
$$

where $0<\imath_{1,1}<\imath_{1,2}<\ldots$ are the positive zeros of $J_{1}^{\prime}$, the 1 st derivative of the Bessel function of order one $J_{1}$ and $\tau=2 r_{\mathrm{c}}^{2} / \sigma^{2}$ is the characteristic time. We note that, as expected, the MSD saturates to $r_{c}^{2}$ in the long-time limit $t \gg \tau$. Then, Equation (57) is the 1 st term of the sum (58) with $a=1, b=8 /\left(l_{11}^{2}\left(1-l_{11}^{2}\right)\right)$ and $c=\iota_{11}^{2}$. Parameters $\sigma$ and $r_{c}$ are unchanged in the two equations (58) and (57).

Diffusion in a potential well. We can state that a particle is attracted by an external force modeled by a potential well $U$. Originally, Kramers [52] introduced such a model for describing 
chemical reactions. His model can be seen as the ( $d$-dimensional) Langevin equation (40) (written here as a SDE) with an extra term depending on $U$

$$
m d v(t)=-\zeta v(t) d t-\nabla U\left(X_{t}\right)+\sqrt{2 \zeta k_{B} T} d B_{t}
$$

where $\nabla$ denotes the gradient operator. Now we make other assumptions on Equation (59) to obtain a process with the MSD (57). First, we suppose that the viscosity is very large, that is the friction coefficient $\zeta$ tends to infinity. Then the acceleration term $m d v(t)$ is negligible. This corresponds to the so-called overdamped condition in physics [34]. The model reduces to

$$
\zeta d X_{t}=-\nabla U\left(X_{t}\right)+\sqrt{2 \zeta k_{B} T} d B_{t}
$$

where $d X_{t}=v(t) d t$. Now, we assume that the potential $U$ is uni-modal; in other words the particle is trapped in a single domain. In this case, $U$ can be approximated by a polynomial of order 2. For simplicity, suppose that the potential is given by the following polynomial:

$$
U\left(x_{1}, \ldots, x_{d}\right)=(1 / 2) \sum_{i=1}^{d} k_{i}\left(x_{i}-\theta_{i}\right)^{2},
$$

where $k_{i}>0, \theta_{i} \in \mathbb{R}$ and $d$ is the dimension of the process. Then the SDE (60) turns into

$$
d X_{t}^{i}=-\lambda_{i}\left(X_{t}^{i}-\theta_{i}\right) d t+\sigma d B_{t}^{i}, \quad i=1, \ldots, d,
$$

where $\sigma=\sqrt{2 k_{B} T \zeta}$ and $\lambda_{i}=k_{i} / \zeta>0$. As in the case of Equation (40), the solution of the SDE (62) is the Ornstein-Uhlenbeck process (different parametrization compared to the SDE (40) with the extra parameters $\theta_{i}$ though). The parameter $k_{i}$ measures the strength of attraction of the potential (related to the potential depth) while $\theta=\left(\theta_{1}, \ldots, \theta_{d}\right)$ is the equilibrium position of the particle. As we already mentioned, the Ornstein-Uhlenbeck is a Gaussian process with normal stationary distribution. In the case of the Ornstein-Uhlenbeck, the mean and covariance of the stationary distribution are

$$
\begin{gathered}
\mathbb{E}\left(\mathrm{X}_{\mathrm{t}}\right)=\theta \\
\operatorname{Cov}\left(\mathrm{X}_{\mathrm{t}}, \mathrm{X}_{\mathrm{s}}\right)=\frac{\sigma^{2}}{2}\left(\begin{array}{ccc}
\left(1-e^{-\lambda_{1}|\mathrm{t}-s|}\right) / \lambda_{1} & & 0 \\
& \ddots & \\
0 & & \left(1-e^{-\lambda_{d}|t-s|}\right) / \lambda_{d}
\end{array}\right) .
\end{gathered}
$$

The MSD of the Ornstein-Uhlenbeck process (62) is given by

$$
\mathbb{E}\left(\left\|X_{t}-X_{0}\right\|^{2}\right)=\sigma^{2}\left(1-e^{-\lambda t}\right) \sum_{i=1}^{d}\left(1 / \lambda_{i}\right),
$$

when $X_{0}$ is drawn with the stationary distribution. When $\lambda_{i}=\lambda$ for $i=1, \ldots, d$ Equation (65) reduces to

$$
\mathbb{E}\left(\left\|X_{t}-X_{0}\right\|^{2}\right)=\frac{d \sigma^{2}\left(1-e^{-\lambda t}\right)}{\lambda} .
$$

Then, we obtain the MSD (57) with $r_{c}^{2}=\sigma^{2} /(2 \lambda), a=2 / d$ and $b=c=1$.

As an example, Hozé [53] studies the postsynaptic AMPAtype glutamate receptor (AMPAR), a protein involved in the fast excitatory synaptic transmission. AMPAR plays a crucial part in many aspects of brain functions including learning, memory and cognition. Aberrant AMPAR trafficking is implicated in neurodegenerative process [54]. Hozé and Holcman [6] use the overdamped Equation (60) with a polynomial of order 2 for the potential $U$ to model potential wells attracting AMPAR in the synapses.

\section{Superdiffusion}

We note that less attention has been paid to superdiffusion in biophysics. We present here the most popular models.

\section{Brownian with drift}

At the macroscopic level, the main type of active intracellular transport involves molecular motors which carry particles (cargo) along microtubular filament tracks (see [4]). The molecular motors and their cargo undergo superdiffusion on a network of microtubules in order to reach a specific area quickly. The molecular motor moves step by step along the microtubules thanks to a mechano-chemical energy transduction process. A single step of the molecular motor is modeled by the socalled Brownian ratchet $[55,56]$. When we observe the motion of the molecular motor along a filament on longer time-scales (several steps), its dynamic can be approximated by a Brownian motion with constant drift (also called directed Brownian) [57, 58]. In specific biological studies (e.g. virus trafficking [59]), the drift term can be calibrated from local interactions with environment, including cytoplasmic intermediate filaments, actin filaments [60] and microtubule network $[59,61]$, which compose the cytoskeleton.

The Brownian motion with drift is solution of the SDE

$$
d X_{t}^{i}=v_{i} d t+\sigma d B_{t}^{1 / 2, i}, \quad i=1, \ldots, d,
$$

where $v=\left(v_{1}, \ldots, v_{d}\right) \in \mathbb{R}^{d}$ is the constant drift parameter modeling the velocity of the molecular motor. Then the MSD of the directed Brownian motion is given by

$$
\mathbb{E}\left(\left\|X_{t}-X_{0}\right\|^{2}\right)=\|v\|_{2}^{2} t^{2}+d \sigma^{2} t
$$

the linear component coming from the Brownian part while the quadratic part is due to the constant drift. In absence of the Brownian component the MSD is quadratic, the motion is described as ballistic that is the particle goes straight.

\section{Anomalous superdiffusion}

Anomalous superdiffusions are the analogue to anomalous subdiffusion. Then the MSD of an 'anomalous superdiffusion' $\left(X_{t}\right)$ is characterized by an MSD which is proportional to the monome $t^{\beta}$,

$$
\mathbb{E}\left(\left\|X_{t}-X_{0}\right\|^{2}\right) \propto t^{\beta},
$$

with $1<\beta<2$.

Fractional Brownian motion. Superdiffusion can also be modeled by the $\mathrm{fBm}$ with Hurst parameter $1 / 2<\mathfrak{h}<1$. In fact, we know that the MSD of the $\mathrm{fBm}$ is given by Equation (69). However, we note that in biophysics the use of the $\mathrm{fBm}$ is mainly related to subdiffusion. 
Lévy walks. In this section the analytical form of the MSD is given in one dimension only, the theory of Lévy walks being essentially developed for one-dimensional problems. We note that Zaburdaev et al. [62] state that the analysis can be formally generalized to higher dimensions but raising some modeling questions. First we must emphasize the difference between Lévy flights and Lévy walks. The Lévy flight consists in a random walk in which the displacements $r$ are characterized by a power-fat tail distribution:

$$
\lim _{|r| \rightarrow \infty} p(r) \propto|r|^{-1-\alpha}, 0<\alpha<2 .
$$

Very large displacements are likely to occur and more specifically, the 2nd order moment of such distribution diverges. Depending on the definition of Lévy flights, some resting times can occur between each jump. In any case, the divergence of the 2nd order moment of a single displacement leads to the divergence of the MSD [63, Chapter 8]. The interest in Lévy flights arose from Lévy's theory that states that the sum of these random independent displacements (equivalently the last position of the particle) obeys a particular distribution as the number of jumps tends to infinity. This property is the counterpart of the central limit theorem when random variables do no exhibit a finite 2nd order moment [62]. However, the divergence of the 2nd order moment does not match with experimental observations [62]. To overcome this difficulty, the step lengths must depend on the time in order to penalize long step lengths by higher time costs [63, Chapter 8]. Now, we define the simplest example of Lévy walk. During one step of the walk, the particle travels at a constant speed $v$ during a random time $t$ before changing direction. In this model, the speed $v$ is constant all along the walk. Then the distance travelled in one step is $|v| t$ and depends on the time. Now the displacement $|r|$ over a period $T$ is bounded by $|v| T$ solving the issue of the infinite 2nd order moment of displacements in the original Lévy flight. We connect Lévy flights and Lévy walks by considering that $\psi(t)$ has a power-fat tail distribution (Equation (70)). Then this process can be seen as a walker traveling at a constant speed $v$ between two locations visited by a Lévy flight [63, Chapter 8]. When $\psi(t)$ is the power law the MSD is (see [64])

$$
\lim _{t \rightarrow \infty} \mathbb{E}\left(\left|X_{t}-X_{0}\right|^{2}\right)=t^{3-\alpha} \text { for } 1<\alpha<2,
$$

defining an anomalous superdiffusion. For extreme values of $\alpha$ the MSD is asymptotically the same as either ballistic motion ( $\alpha<1$ heavy tails for $\psi(t)$ leading to few switches of direction) or Brownian motion $(\alpha>2$ small tails for $\psi(t)$ leading to frequent switches of direction). Another model includes random rests at each turning points generated by a distribution $\psi_{r}(t)$. Klafter and Sokolov [63, Chapter 8] assume that $\psi(t)$ (respectively $\left.\psi_{r}(t)\right)$ has power-fat tail distribution (see Equation (70)) of parameter $\alpha$ (respectively $\gamma$ ). Then the competition between parameters $\alpha$ and $\gamma$ leads to different types of diffusion including anomalous superdiffusion or Brownian motion asymptotically. Finally, Zaburdaev et al. [65] propose a model with random velocities which have a power law distribution. Again, the competition between the distribution of velocity and $\psi(t)$ lead to different types of diffusions including anomalous superdiffusion.

In recent experimental studies and modeling [66, 67], the authors observed that active cargo transport tends to self-organize into Lévy walks. More specifically it has been established that the biomolecule moves along a microtubule driven by motors with a global constant velocity for some random time and then detach from the microtubule and reattaches to a new microtubule moving along another direction [62]. Accordingly, Chen et al. [66] first defined the concept of memoryless self-reinforced directionality, which is helpful to justify the emergence of Lévy walks. In [67, 68], the authors recently studied this related non-Markovian transport of biomolecules (e.g. vesicles in RPE cells) and proposed a promising theoretical model involving non-Markovian detachment rate and superdiffusive Lévy-walk-like cargo movement.

\section{Conclusion}

In this paper, we presented three main classes of diffusions, namely Brownian motion, subdiffusion and superdiffusion, which are tractable microscopic and macroscopic models of biomolecule transport inside eukariotic cells. The diffusion phenomenon, described by Robert Brown in the early 19th, is mainly due to the thermal agitation in the medium. This agitation results from shocks between molecules and induces stochastic effects. For each diffusion type, we gave examples of models used in biophysics. Typically, we focused on the Ornstein-Uhlenbeck process and the $\mathrm{fBm}$ (with $0<\mathfrak{h}<1 / 2$ ) for modeling subdiffusion. We used the Brownian with drift and the $\mathrm{fBm}$ (with $1 / 2<\mathfrak{h}<1$ ) for modeling superdiffusion. However, there exists a wide variety of models for subdiffusion and superdiffusion. We emphasized that, in biophysics, some processes are considered as subdiffusive or superdiffusive even if there are not diffusions according to the probabilistic definition. As an example, CTRWs are not diffusions since their paths are not continuous. We gave mathematically detailed formulas and define the classification through the MSD criterion.

Meanwhile, an important challenge is to estimate model parameters [6] from image data obtained with experimental microscopy techniques [28] such as fluorescence correlation spectroscopy and fluorescence recovery after photobleaching and to classify tracks computed with dedicated algorithms [69]. In [3], we defined several test procedures to classify the observed trajectories into the three diffusion types. Another issue is to simulate more sophisticated models as presented in $[36,56,70$, 71 , including multi-scale models to take into account complex interactions, signaling pathways, properties of the cytoskeleton and of the viscoelastic cytosol of living cells [56], geometry of the cell and interaction with neighboring cells. If the dynamics of particles is governed by diffusions as presented in this paper, we should be able to reproduce the observed spatial patterns by simulations. However, here we presented models for describing individual particle motion, an approach which does not take into account interactions between particles. Combining models of individual dynamics and particle interactions should allow one to capture the complexity of the intracellular biological processes. Then agent-based modeling [72-74] allows a realistic simulation of a system of interacting diffusive particles with heterogeneous constraints, including interactions with actin filaments and microtubules, cell organelles, cell geometry, cytoplasm viscosity, molecular affinity... This promising approach was used to investigate filopodia-lamellipodia interface [72], dynamics of protein aggragates in E. coli [73], enzyme kinetics [74], but is also known to be computationally demanding. New efforts are then required to be able to mimic realistic phenomena with reduced computation time. 


\section{Key points}

- We present an overview of diffusion models commonly used for quantifying the dynamics of intracellular particles (e.g. biomolecules) inside living cells.

- This paper summarizes the mathematical definitions of superdiffusion, free diffusion and subdiffusion, wellgrounded in Einstein's and Langevin's theories.

- Applications of diffusion models include protein trafficking and transport, and membrane diffusion.

\section{Supplementary Data}

Supplementary data are available online at https://academic. oup.com/bib.

\section{Funding}

This work was supported by Inria and CREST-ENSAI. This work was also supported by the French National Research Agency (France-BioImaging infrastructure-ANR-10-INBS-04, DALLISH-ANR-16-CE23-0005).

\section{References}

1. Qian H, Sheetz MP, Elson EL. Single particle tracking. Analysis of diffusion and flow in two-dimensional systems. Biophys J 1991;60:910.

2. Saxton MJ, Jacobson K. Single-particle tracking: applications to membrane dynamics. Annu Reu Biophys Biomol Struct 1997; 26:373-99.

3. Briane V, Kervrann C, Vimond M. Statistical analysis of particle trajectories in living cells. Phys Rev E 2018; 97: 062121.

4. Goychuk I, Kharchenko VO, Metzler R. Molecular motors pulling cargos in the viscoelastic cytosol: power strokes beat subdiffusion. Phys Chem Chem Phys 2014;16:16524-35.

5. Metzler R, Klafter J. The random walk's guide to anomalous diffusion: a fractional dynamics approach. Phys Rep 2000; 339:1-77.

6. Hozé N, Holcman D. Statistical methods for large ensembles of super-resolution stochastic single particle trajectories in cell biology. Annu Rev Stat Appl 2017;4:189-223.

7. Saxton MJ. Anomalous diffusion due to obstacles: a Monte Carlo study. Biophys J 1994;66:394.

8. Berry $\mathrm{H}$, Chaté $\mathrm{H}$. Anomalous diffusion due to hindering by mobile obstacles undergoing brownian motion or OrnsteinUlhenbeck processes. Phys Reu E 2014;89:022708.

9. Goychuk I. Viscoelastic subdiffusion: from anomalous to normal. Phys Rev E 2009;80:046125.

10. Feder TJ, Brust-Mascher I, Slattery JP, et al. Constrained diffusion or immobile fraction on cell surfaces: a new interpretation. Biophys J 1996;70:2767.

11. Tejedor V, Bénichou O, Voituriez R, et al. Quantitative analysis of single particle trajectories: mean maximal excursion method. Biophys J 2010;98:1364-72.

12. Gal N, Lechtman-GoldsteinD WD. Particle tracking in living cells: a review of the mean square displacement method and beyond. Rheologica Acta 2013;52:425-43.
13. Lund FW, Jensen MLV, Christensen T, et al. Spattrack: an imaging toolbox for analysis of vesicle motility and distribution in living cells. Traffic 2014;15:1406-29.

14. Lysy M, Pillai NS, Hill DB, et al. Model comparison and assessment for single particle tracking in biological fluids. J Am Stat Assoc 2016;111:1413-26.

15. Michalet X. Mean square displacement analysis of singleparticle trajectories with localization error: Brownian motion in an isotropic medium. Phys Rev E 2010; 82:041914.

16. Pisarev AS, Rukolaine SA, Samsonov AM, et al. Numerical analysis of particle trajectories in living cells under uncertainty conditions. Biophysics 2015;60:810-7.

17. Monnier N, Guo SM, Mori M, et al. Bayesian approach to msdbased analysis of particle motion in live cells. Biophys J 2012; 103:616-26.

18. Meroz Y, Sokolov IM. A toolbox for determining subdiffusive mechanisms. Phys Rep 2015;573:1-29.

19. Karlin S. A Second Course in Stochasic Processes. San Diego, New York, Boston, London, Sydney, Tokyo, Toronto: Academic Press, 1981.

20. Klebaner FC. Introduction to Stochastic Calculus with Applications. London: Imperial College Press, 2012.

21. Mandelbrot BB, Van JW. Fractional brownian motions, fractional noises and applications. SIAM Rev 1968;10:422-37.

22. Gallardo L. Mouvement brownien et calcul d'Itô: Cours et Exercices corrigés. Hermann, 2008.

23. Kolmogorov AN. The local structure of turbulence in incompressible viscous fluid for very large Reynolds numbers. Dokl Akad Nauk SSSR 1941;30:299-303.

24. Hurst HE. Long-term storage capacity of reservoirs. Trans Amer Soc Civil Eng 1951;116:770-808.

25. Decreusefond L, Ustünel AS. Stochastic analysis of the fractional brownian motion. Potential Anal 1999;10: 177-214.

26. Coutin L, Qian Z. Stochastic analysis, rough path analysis and fractional brownian motions. Probab Theory Relat Fields 2002;122:108-40.

27. Einstein A. On the motion of small particles suspended in liquids at rest required by the molecular-kinetic theory of heat. Ann Phys 1905;17:549-60.

28. Höfling F, Franosch T. Anomalous transport in the crowded world of biological cells. Rep Prog Phys 2013;76:046602.

29. Fick A. V. on liquid diffusion. Philos Mag Ser 4 1855;10:30-9.

30. Langevin P. Sur la théorie du mouvement brownien. C R Acad Sci Paris 1908;146:530.

31. Kou SC. Stochastic modeling in nanoscale biophysics: subdiffusion within proteins. Ann Appl Stat 2008; 2:501-35.

32. Schuss Z. Theory and Applications of Stochastic Processes: An Analytical Approach, Vol. 170. New York, Dordrecht, Heidelberg, London: Springer Science \& Business Media, 2009.

33. Uhlenbeck GE, Ornstein LS. On the theory of the brownian motion. Phys Rev 1930;36:823.

34. Van Kampen NG. Stochastic Processes in Physics and Chemistry, Vol. 1. Oxford: Elsevier, 1992.

35. Waterston JJ, Rayleigh L. On the physics of media that are composed of free and perfectly elastic molecules in a state of motion. Philos Trans R Soc Lond A 1892;183:1-79.

36. Bressloff PC. Stochastic Processes in Cell Biology, Vol. 41. Cham, Heidelberg, New York, Dordrecht, London: Springer, 2014.

37. Sokolov IM. Models and anomalous diffusion in crowded environments. Soft Matter 2012;8:9043-52. 
38. Jeon JH, Tejedor V, Burov S, et al. In vivo anomalous diffusion and weak ergodicity breaking of lipid granules. Phys Rev Lett 2011;106:048103.

39. Weber SC, Spakowitz AJ, Theriot JA. Bacterial chromosomal loci move subdiffusively through a viscoelastic cytoplasm. Phys Rev Lett 2010;104:238102.

40. Zwanzig R. Nonequilibrium statistical mechanics. Oxford University Press, 2001.

41. Chandler D. Introduction to modern statistical mechanics. Oxford University Press, 1987.

42. Scher H, Montroll EW. Anomalous transit-time dispersion in amorphous solids. Phys Rev B 1975;12:2455.

43. Zhizhina E, Komech S, Descombes X. Modelling axon growing using CTRW. arXiv preprint arXiv:1512.02603, 2015.

44. Havlin S, Ben-Avraham D. Diffusion in disordered media. Adv Phys 1987;36:695-798.

45. Dix JA, Verkman AS. Crowding effects on diffusion in solutions and cells. Annu Rev Biophys 2008;37:247-63.

46. Holcman D, Hozé N, Schuss Z. Narrow escape through a funnel and effective diffusion on a crowded membrane. Phys Rev E 2011;84:021906.

47. Grassberger P. Conductivity exponent and backbone dimension in 2-D percolation. Physica A 1999;262: 251-63.

48. Kusumi A, Nakada C, Ritchie K, et al. Paradigm shift of the plasma membrane concept from the two-dimensional continuum fluid to the partitioned fluid: high-speed singlemolecule tracking of membrane molecules. Annu Rev Biophys Biomol Struct 2005;34:351-78.

49. Kusumi A, Sako Y, Yamamoto M. Confined lateral diffusion of membrane receptors as studied by single particle tracking (nanovid microscopy). Effects of calcium-induced differentiation in cultured epithelial cells. Biophys J 1993;65:2021.

50. Saxton MJ. Lateral diffusion in an archipelago. Singleparticle diffusion. Biophys J 1993;64:1766-80.

51. Bickel T. A note on confined diffusion. Physica A 2007; 377:24-32.

52. Kramers HA. Brownian motion in a field of force and the diffusion model of chemical reactions. Phys Ther 1940; 7:284-304.

53. Hozé N. Modélisation et méthodes d'analyse de la diffusion et agrégation au niveau moléculaire pour l'organisation sous-cellulaire. PhD diss., Université Pierre et Marie CurieParis 6, 2013.

54. Henley JM, Barker EA, Glebov OO. Routes, destinations and delays: recent advances in ampa receptor trafficking. Trends Neurosci 2011;34:258-68.

55. Reimann P. Brownian motors: noisy transport far from equilibrium. Phys Rep 2002;361:57-265.

56. Goychuk I, Kharchenko VO, Metzler R. How molecular motors work in the crowded environment of living cells: coexistence and efficiency of normal and anomalous transport. PLoS One 2014;9:e91700.

57. Peskin CS, Oster G. Coordinated hydrolysis explains the mechanical behavior of kinesin. Biophys J 1995;68:202S.

58. Elston TC. A macroscopic description of biomolecular transport. J Math Biol 2000;41:189-206.

59. Lagache T, Holcman D. Effective motion of a virus trafficking inside a biological cell. SIAM J Appl Math 2008;68: 1146-67.

60. Ajdari A. Transport by active filaments. Europhys Lett 1995; 31:69-74

61. Lawley SD, Tuft M, Brooks HA. Coarse-graining intermittent intracellular transport: two-and three-dimensional models. Phys Rev E 2015;92:042709.

62. Zaburdaev V, Denisov S, Klafter J. Lévy walks. Rev Mod Phys 2015;87:483-530.

63. Klafter J, Sokolov IM. First steps in random walks: from tools to applications. Oxford University Press, 2011.

64. Zumofen G, Klafter J. Scale-invariant motion in intermittent chaotic systems. Phys Rev E 1993;47:851.

65. Zaburdaev V, Schmiedeberg M, Stark H. Random walks with random velocities. Phys Rev E 2008;78: 011119.

66. Chen K, Wang B, Granick S. Memoryless self-reinforcing directionality in endosomal active transport within living cells. Nat Mater 2015;14:589.

67. Fedotov S, Korabel N, Waigh TA, et al. Memory effects and Lévy walk dynamics in intracellular transport of cargoes. Phys Rev E 2018;98:042136.

68. Korabel N, Waigh TA, Fedotov S, et al. Non-Markovian intracellular transport with sub-diffusion and runlength dependent detachment rate. PLoS One 2018;13: e0207436.

69. Chenouard N, Smal I, De Chaumont F, et al. Objective comparison of particle tracking methods. Nat Methods 2014; 11:281.

70. Bressloff PC, Newby JM. Stochastic models of intracellular transport. Reu Mod Phys 2013;85:135.

71. Etoc F, Balloul E, Vicario C. Non-specific interactions govern cytosolic diffusion of nanosized objects in mammalian cells. Nat Mater 2018;17:740-6.

72. Azimi M, Jamali Y, Mofrad MRK. Accounting for diffusion in agent based models of reaction-diffusion systems with application to cytoskeletal diffusion. PLoS One 2011; 6: e25306.

73. Coquel A-S, Jaciob J-P, Primet M, et al. Localization of protein aggregation in Escherichia coli is governed by diffusion and nucleoid marcromolecular crowdoing effect. PLoS Comput Biol 2013;9:e1003038.

74. Pérez Rodriguez G, Gameiro D, Pérez-Pérez M, et al. Single molecule simulation of diffusion and enzyme kinetics.J Phys Chem B 2016;120:3809-20. 